\title{
Cryogenic systems in Radio Astronomy
}

\author{
José Manuel Serna Puente ${ }^{1}$ \\ Affiliation: Centro Astronómico de Yebes (OAN-IGN) \\ Address, Country: Yebes-Guadalajara-Spain \\ E-mail: jm.serna@oan.es
}

In this paper the fundamentals of the design of a cryogenic system in radio astronomy are presented. The working principle of a cryostat and the different elements that are necessary for its correct operation are shown. Special attention is paid to the different thermal loads inside the cryostat in order to choose the refrigerator system that allows us to achieve the needed temperatures.

2nd MCCT-SKADS Training School Radio Astronomy: fundamentals and the new instruments

Sigüenza (Spain)

August $26^{\text {th }}-$ September $4^{\text {th }} 2008$

\footnotetext{
${ }^{1}$ Speaker
} 


\section{Introduction}

The first elements in the signal path of a radio telescope contribute the greatest amount to the system noise temperature, and for this reason radio astronomy receivers are generally operated at cryogenic temperatures.

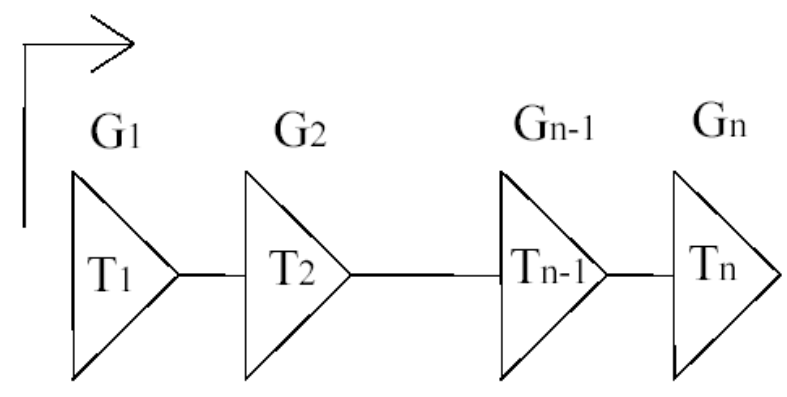

Figure 1: Noise temperature of a receiver chain

$$
T_{e}=T_{1}+\frac{T_{2}}{G_{1}}+\frac{T_{3}}{G_{1} \cdot G_{2}}+\ldots+\frac{T_{N}}{G_{1} \cdot G_{2} \cdots G_{N-1}}
$$

In order to reach cryogenic temperatures, a vacuum chamber (dewar) containing the receiver is evacuated to a very high vacuum, and a close-cycle refrigerator is used to remove the heat.

Thus, the cryogenic system is a crucial element governing a receiver's performance. When designing cryogenic systems, the designer is faced with conflicting requirements which require careful analysis to achieve optimum results.

It is important to know that, depending on the system, inside the cryostat we are going to have different elements of the receiver chain:

- LNA (Low Noise Amplifiers).

- Directional couplers.

- Polarizers.

- Corrugated horn.

- Cables (DC and RF).

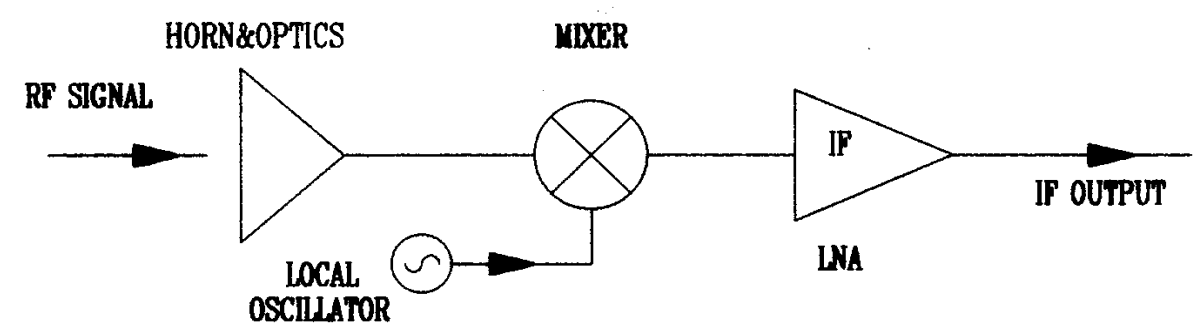

Figure 2: Typical receiver chain 
Among the most important considerations are the performance of the refrigerator (temperature reached and maintained, degree of vacuum achieved). Other important considerations in the performance of cryogenic systems are reliability and maintainability. Refrigerator selection, choice of materials for the vacuum chamber walls and internal components, fabrication techniques, cleaning procedures and evacuation procedures are important considerations affecting reliability.

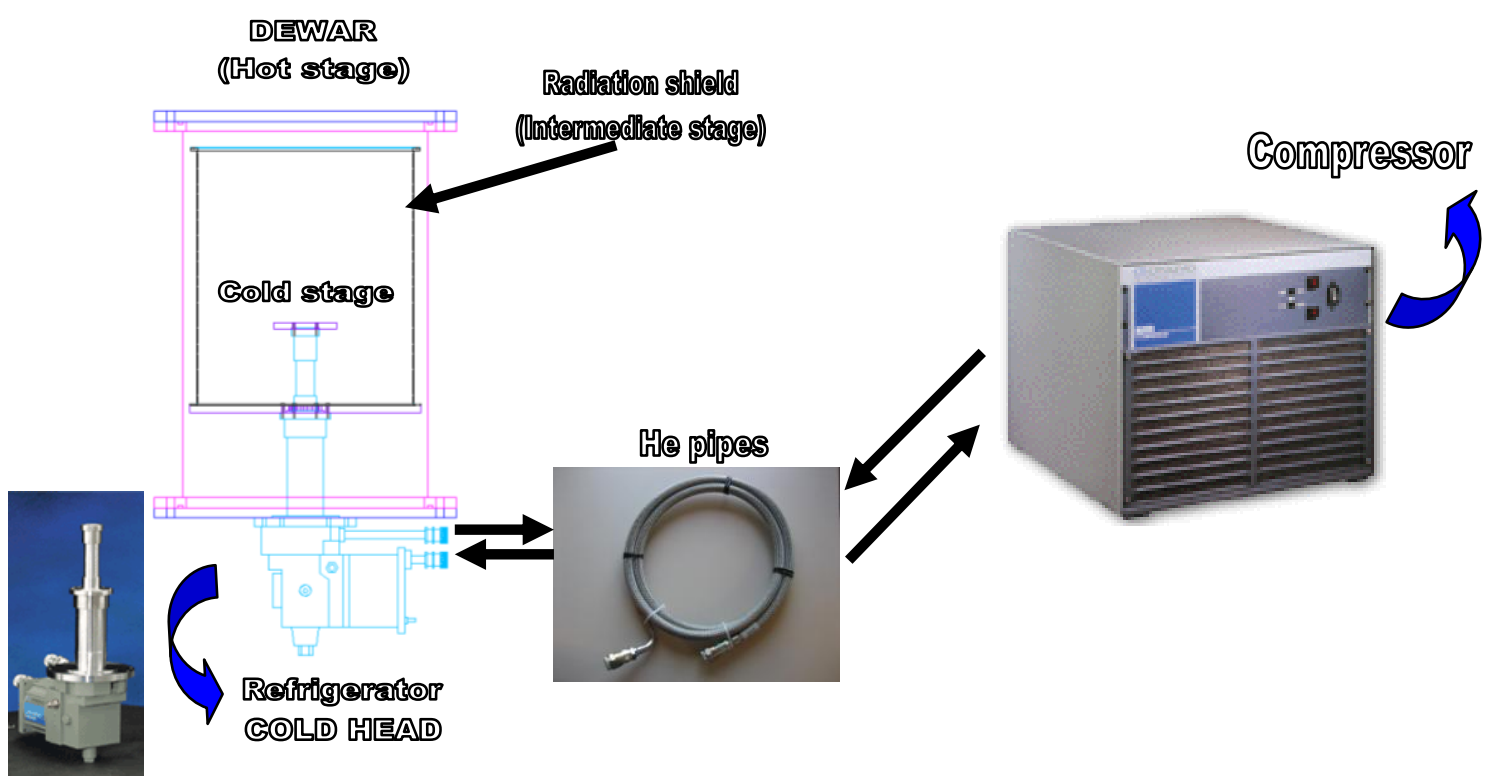

Figure 3: Cryogenic system scheme

There are different types of cryostats depending on the working principle:

- Closed cycle: Using "cold heads" (He gas, $77 \mathrm{~K}$ and $20 \mathrm{~K}$ ).

- Open cycle: (Liquid $\mathrm{N} \rightarrow 77 \mathrm{~K}$, liquid $\mathrm{He} \rightarrow 4 \mathrm{~K}$ ).

- Hybrid: "cold head" + liquid He filling $\rightarrow 0.35 \mathrm{~K}$ with $\mathrm{He}-3$.

In the following figure, the interior of the $22 \mathrm{GHz}$ receiver cryostat in Yebes is Shown:

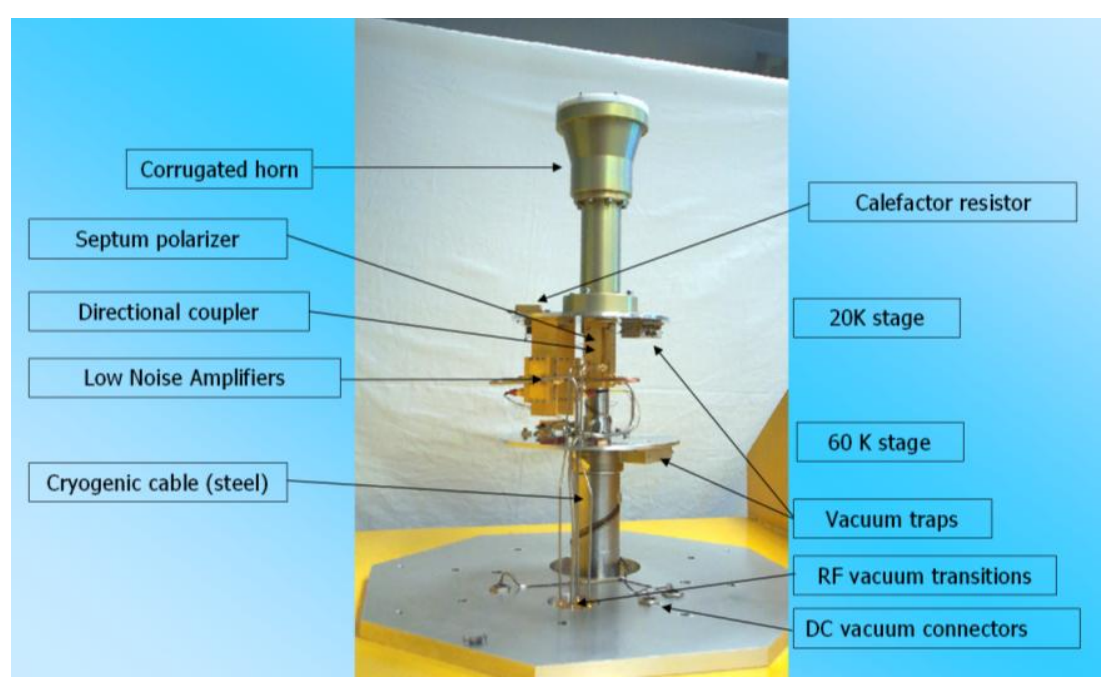

Figure 4: The interior of a cryostat 


\section{Thermal loads.}

\subsection{Load due to residual gas.}

$$
a_{0}=\frac{\text { actual_energy_transfer }}{\text { maximum_possible_energy_transfer }}
$$

$\mathrm{K} 1 \rightarrow$ Constant depending on the gas (air:1,2).

$\mathrm{A} \rightarrow$ Cold stage area.

a0 $\rightarrow$ Accommodation coefficient (realted with the degree of approach of the molecules to the thermal equilibrium). Worst case: $\mathrm{a} 0=1$.

$\mathrm{P} \rightarrow$ Pressure inside the cryostat $(\mathrm{Pa})$.

T2 $\rightarrow$ Hot stage temperature.

$\mathrm{T} 1 \rightarrow$ Cold stage temperature.

In order to reduce this component it is important to reduce the pressure inside the cryostat. In $20 \mathrm{~K}$ closed cycle cryostats it is necessary to pump below $10^{-3} \mathrm{mbar}$. In $4 \mathrm{~K}$ hybrid cryostats $10^{-5} \mathrm{mbar}$ pressure is needed.

$$
\begin{aligned}
& 1 P a=7.5 \cdot 10^{-3} \text { torr } \\
& 1 \text { torr }=1.33 \text { mbar }
\end{aligned}
$$

\subsection{Load due to solid conduction.}

$$
W_{\text {cond }}=\frac{A}{l} \cdot \lambda \cdot\left(T_{2}-T_{1}\right)
$$

A $\rightarrow$ Cross section area of the conducting element.

$\mathrm{L} \rightarrow$ Conducting element length.

$\lambda \rightarrow$ Thermal conductivity of the material (intermediate between T2 and T1).

T2 $\rightarrow$ Hot stage temperature.

T1 $\rightarrow$ Cold stage temperature.

In order to reduce this component it is important to use materials with low thermal conductivity, a small cross section and length.

\subsection{Load due to radiation.}

$$
\begin{gathered}
W_{\text {rad }}=F_{e} \cdot F F \cdot \sigma \cdot A_{1} \cdot\left(T_{2}^{4}-T_{1}^{4}\right) \\
F_{e}=\frac{\varepsilon_{1} \cdot \varepsilon_{2}}{\varepsilon_{2}+\left(A_{1} / A_{2}\right) \cdot\left(1-\varepsilon_{2}\right) \cdot \varepsilon_{1}}
\end{gathered}
$$

$\mathrm{Fe} \rightarrow$ Emissivity factor. 
$\mathrm{FF} \rightarrow$ Configuration factor (depends on the geometry).

$\sigma \rightarrow$ Stefan-Boltzman constant $=5.67 \times 10-8 \mathrm{Wm}-2 \mathrm{~K}-4$

A1 $\rightarrow$ Area of inner surface.

A2 $\rightarrow$ Area of outer surface.

T2 $\rightarrow$ Hot stage temperature.

$\mathrm{T} 1 \rightarrow$ Cold stage temperature.

$\varepsilon 1, \varepsilon 2 \rightarrow$ emissivity of the surfaces.

In order to reduce this component it is important to use low emissivity materials and, because of the great dependence with temperature, employ special techniques like MLI. The first method to reduce the radiative load is to clean and polish the surfaces to reduce the emissivity.

It is also important to have an intermediate stage because of the great dependence with the temperature.

\subsubsection{Multi Layer Insulation (MLI).}

Multilayer insulations consist of alternating layers of highly reflecting materials (aluminum) and low-conductivity spacer (mylar plastic-polyester film).

The sheets of aluminized mylar are crinkled in order to reduce the contact surface between layers.

Multilayer insulations must be evacuated to pressures below $10 \mathrm{mPa}$ $\left(\sim \mathbf{1 0}^{-4} \mathbf{m b a r}\right)$ to be effective.

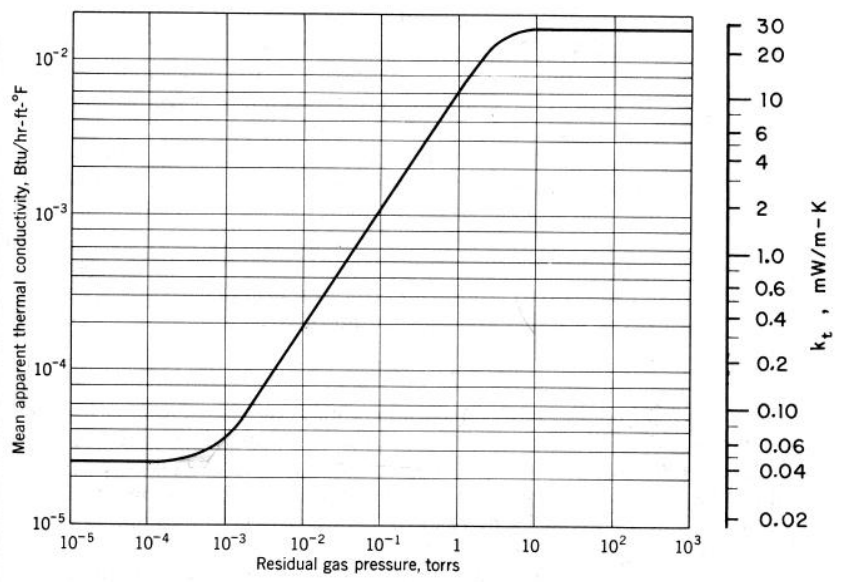

Figure 5: MLI behavior versus pressure

The density of layers must be carefully selected because if the insulation is compressed too tighly, solid conduction begins to increase and the behaviour of the MLI is not the appropriate. 


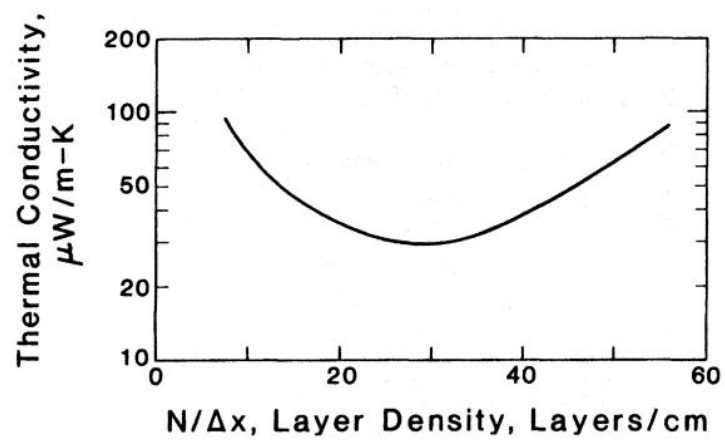

Figure 6: MLI layer density

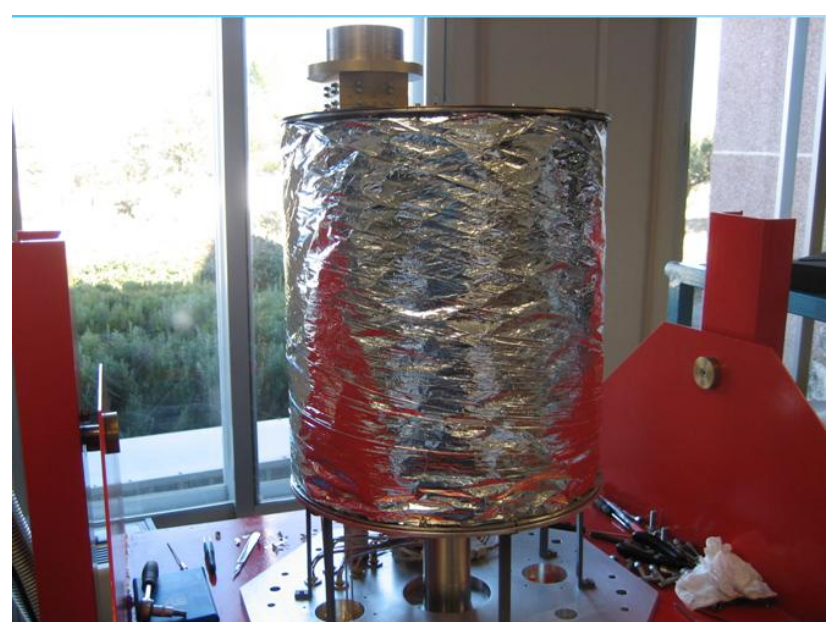

Figure 7: Example of MLI in an intermediate stage of a cryostat

\subsection{Load due to power dissipation in active devices.}

The Low Noise Amplifiers are always installed directly in the COLD stage. It is important to take into account the effect of the LNA power dissipation.

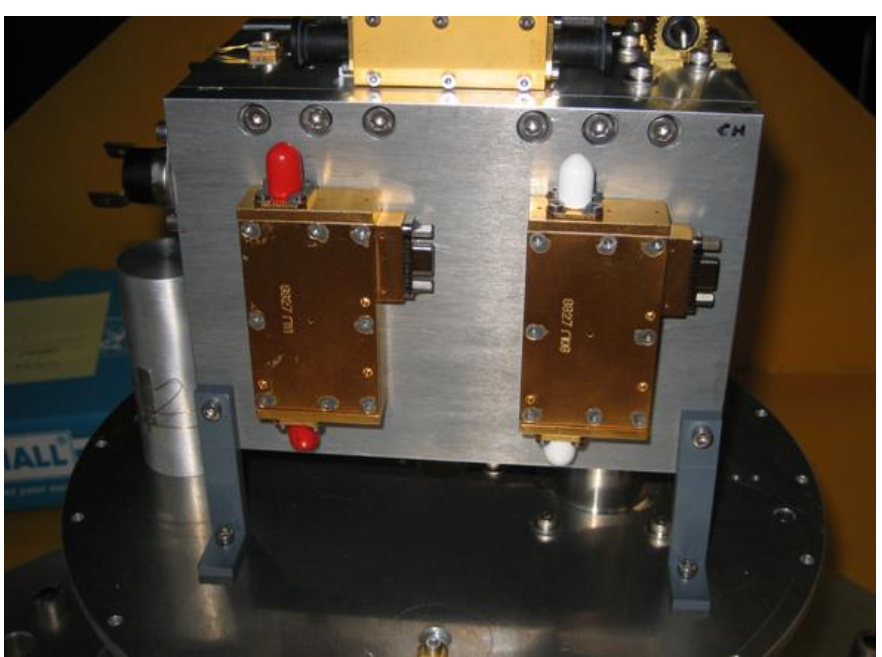

Figure 8: LNAs installed in the cold stage 


\section{Refrigerator selection.}

The most important parameter of a refrigerator is its refrigerator capacity.

To determine the capacity of the refrigerator required, the system designer must estimate both the conductive and radiation loading to be absorbed by the refrigerator cold head at both the intermediate and cold stages.

A third heat load is due to the heat conducted from the dewar walls to the cold head by the residual gas inside the dewar (convection). This third component of heat load is negligible when the vacuum quality is good. Hence, the importance of obtaining high vacuum quality should be stressed during the design and operation of the system.

Finally, any power dissipation due to active devices should be added.

$$
\mathrm{W}_{\mathrm{TOT}}=\mathrm{W}_{\text {cond }}+\mathrm{W}_{\text {rad }}+\mathrm{W}_{\mathrm{gas}}+\mathrm{W}_{\mathrm{dis}}
$$

Once the thermal load estimations are made for each stage of the refrigerator, these values may be plotted on the load curves supplied by the manufacturer.

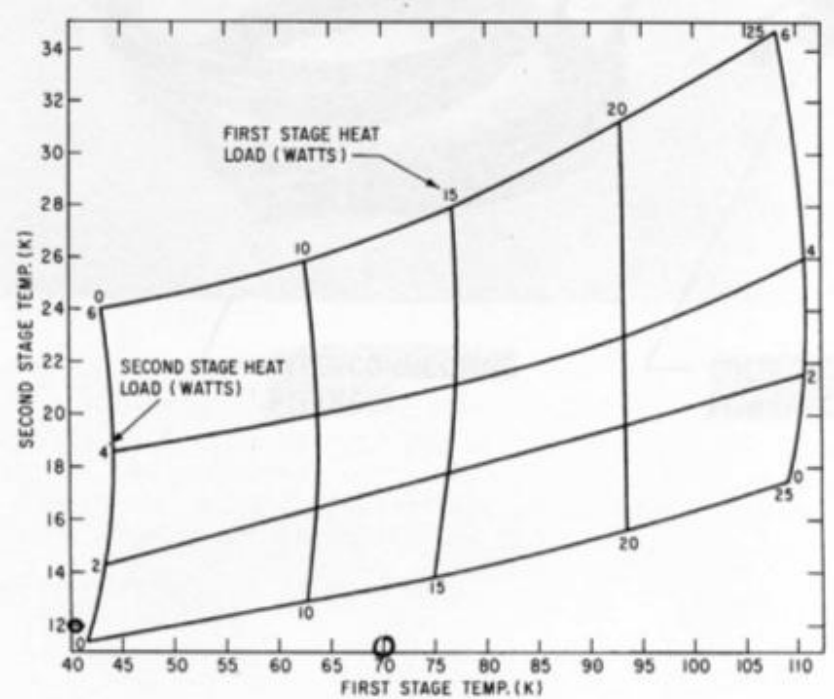

Figure 9: Refrigerator capacity of the Cold Head CTI-350 (50Hz)

\section{References}

[1] “Cryogenic Heat Transfer”. Randall F.Barron. Taylor and Francis, 1999.

[2] "Cryogenic Systems". Randall F.Barron. Oxford Science publications, 1985.

[3] "Experimental Techniques in Low-Temperature Physics". Guy K. White.

[4] "Guidelines for the design of cryogenic systems". NRAO.

[5] "Cryogenic Process Engineering." Klaus D. Timmerhaus, Thomas M. Flynn.

[6] "Cryogenic engineering". Hands.

[7] "Handbook of cryogenic engineering". Weidend.

[8] "CTI-Cryogenics cryodyne refrigeration systems". 